\title{
Nailfold Capillaroscopy: An Important Clinical Tool for the Vascular Surgeon
}

Tracy M Frech ${ }^{1 *}$, Brian Breviu ${ }^{2}$, Jessica T Zhu ${ }^{3}$ and Lesley Saketkoo ${ }^{4}$

${ }^{1}$ University of Utah Department of Internal Medicine, Division of Rheumatology and Salt Lake City Veterans Affair Medical Center, USA

${ }^{2}$ University of Utah Department of Internal Medicine and Salt Lake City Veterans Affair Medical Center, USA

${ }^{3}$ Undergraduate Boston College, Biology B.S., USA

${ }^{4}$ Louisiana State University Health Science Center, Department of Internal Medicine, Division of Rheumatology, USA

\begin{abstract}
Purpose of review: Nailfold capillary microscopy or capillaroscopy is an established clinical exam procedure for dermatologists and rheumatologists. It is essential in the examination of patients with Raynaud's phenomenon.

Findings: A history of Raynaud's phenomenon is an important symptom for vascular surgeons to elicit from patients presenting with digital ulcerations. If a history of Raynaud's phenomenon is present then Nailfold capillaroscopy is an essential clinical exam skill to perform.
\end{abstract}

Summary: In this review, the applicability of capillaroscopy for personalized medicine to direct cost effective laboratory screening for vascular surgeons assessing patients with Raynaud's phenomenon is discussed.

Keywords: Raynaud's phenomenon; Capillaroscopy; Digital ulcer; Connective tissue disease

\section{Introduction}

The concept of personalized medicine focuses on bringing mechanism-based diagnostics and therapeutics to an individual in order to guide interventions to impact disease risk, prevention, prognosis, and cure [1]. It is a concept that must balance the deluge of "omic" information with practical clinical applications, while minimizing health care costs. Often the vascular surgeon is confronted by a concern underlying vasculopathy when a patient reports the presence of a history of Raynaud's phenomenon, particularly in the setting of an ischemic digit. While it may be tempting to send an "autoimmune laboratory panel" in such a patient, microscopy of the nailfold capillaries or Nailfold capillaroscopy is the first step for evaluating a patient with Raynaud's phenomenon. This clinical examination skill may have a transformative impact on disease prediction and is an important skill for vascular surgeons.

In dermatology and rheumatology nailfold capillaroscopy is an important diagnostic tool to distinguish between primary and secondary Raynaud's phenomenon. As such, eliciting a history of Raynaud's phenomenon and performing capillaroscopy is equally important to vascular surgeons, especially when a patient presents with a digital ulceration. Raynaud's phenomenon is a phasic event of the distal part of digits in which arteries, pre-capillary arterioles, and cutaneous arteriovenous shunts become constricted and ischemic

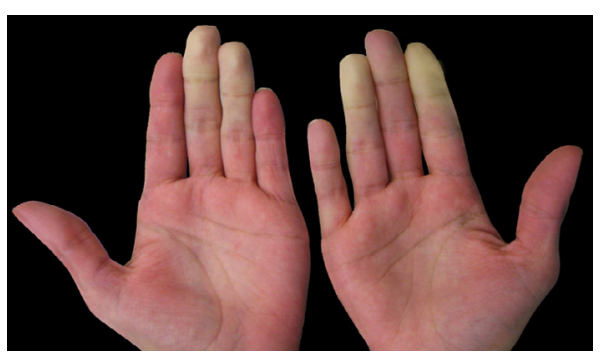

Figure 1: Raynaud's Phenomenon. (represented by demarcated pale and cyanotic skin) followed by a rapid reflow of blood to the digits (reactive hyperemia) primarily in response to cold temperature (Figure 1)[1]. Raynaud's phenomenon is an exaggeration of the physiological response to cold and/or stressful environment. The attacks are usually associated with paresthesias and ischemic pain, and thus are symptomatically important for a vascular surgeon to appreciate. Importantly, nailfold capillaroscopy has been suggested to reflect pathology in other microvascular organs.

\section{Raynaud's Phenomenon}

The pathogenesis of Raynaud's phenomenon essentially involves the complex relationship between vascular endothelium, smooth muscle cells, and nerve terminals. While the specific interactions between these cell types within the vascular microenvironment determines the severity of the Raynaud's phenomenon, all Raynaud's phenomenon is thought to represent a perturbation of the balance between vasoconstriction and vasodilatation in digital blood vessels [2].

Primary or idiopathic Raynaud's phenomenon is seen in up to $10 \%$ of the general population. It more commonly affects women ages 15 to 30 , is generally mild, involves the digits symmetrically, and is sometimes familial [3-5]. While it is symmetric, certain digits may be more severely affected. In the setting of an underlying connective tissue disease, termed secondary Raynaud's phenomenon or Raynaud's disease, the severity and duration of the vasospastic response can result in tissue necrosis with resultant digital ulceration and gangrene (Figure 2). These patients tend to present at an age greater than 30 years and have more intense episodes than those with the primary form.

*Corresponding author: Tracy M Frech, University of Utah Department of Internal Medicine, Division of Rheumatology and Salt Lake City Veterans Affair Medical Center, USA, Tel: 801581 4333; E-mail: Tracy.Frech@hsc.utah.edu

Received July 22, 2014; Accepted August 20, 2014; Published August 22, 2014

Citation: Frech TM, Breviu B, Zhu JT, Saketkoo L (2014) Nailfold Capillaroscopy: An Important Clinical Tool for the Vascular Surgeon. J Vasc Med Surg 2: 152 doi: 10.4172/2329-6925.1000152

Copyright: @ 2014 Frech TM, et al. This is an open-access article distributed unde the terms of the Creative Commons Attribution License, which permits unrestricted use, distribution, and reproduction in any medium, provided the original author and source are credited. 


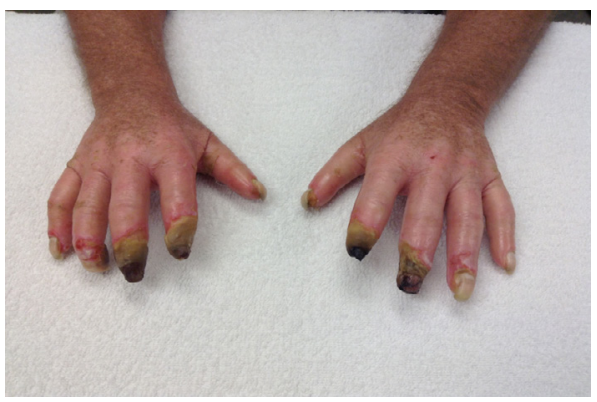

Figure 2: Digital Ischemia.

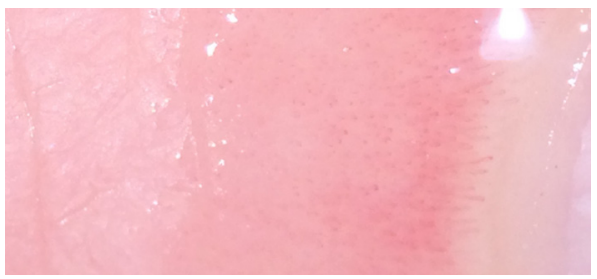

Figure 3: Normal Capillary Pattern.

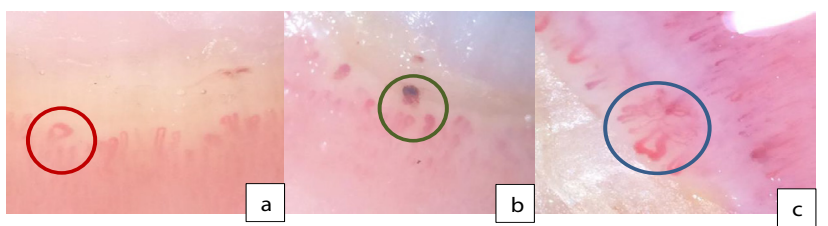

Figure 4: Capillaroscopy Abnormalities: (a) Early changes with dilated loop (red circle); (b) Active change with micro-hemorrhage (green circle); (c) Late change with micro-hemorrhage vessel drop out and neoangiogenesis (blue circle).

The classification of Raynaud's phenomenon as primary or secondary is important. Since Raynaud's phenomenon is the most common and earliest manifestation of Systemic sclerosis (SSc) and is also common in Systemic Lupus Erythematosus (SLE), Sjogren's syndrome, and dermatomyositis, the most important aspect of an assessment of a patient presenting with Raynaud's phenomenon is determining whether it is primary or secondary. Proper identification of an underlying connective tissue disease will allow for the prevention of possible critical ischemia.

\section{Nailfold Capillaroscopy}

Nailfold capillaroscopy is a safe, non-invasive tool to morphologically study the microcirculation that is critical to differentiate primary Raynaud's phenomenon from secondary Raynauds phenomenon, which is related to a connective tissue disease. Nailfold capillaroscopy evaluates the architecture of the skin capillaries of the fingers, where the capillaries run parallel to the skin surface. This procedure evaluates the number, density and the appearance of parallel hairpin-like capillary architecture of the skin capillaries of the fingers.

This clinical exam finding is detected by using light microscopy to evaluate the nailfolds of eight fingers, excluding the thumbs. Capillaroscopy is performed at room temperature after 15-20 minutes of acclimatization in a sitting position [6]. Immersion oil is placed on each nailfold to improve visibility. While rapid differentiation between a healthy and a pathologic pattern can be achieved by a 10fold magnification with a dermatoscope, various devices with low (20x) to high (200-600x) magnification are available to improve detection of enlargement, bleeding, and loss of capillaries [6]. Depending on institutionally available equipment, a dermatoscope, USB digital microscope, stereomicroscope, or video-capillaroscope equipment, can be used to assess the density (number) and morphology of capillaries in a one-millimeter $(\mathrm{mm})$ visual field. An ophthalmoscope (with the lens set at 20 diopters or higher for better resolution) or a modified dermatoscope can be used in the primary care setting if a video capillarscopy or stereomicroscope is not available [7].

A normal capillaroscopic pattern is characterized between 9 to 14 homogenously distributed hairpin-shaped capillaries in one $\mathrm{mm}$ (Figure 3). Capillaroscopy is able to identify subtle features unique to a diagnosis of connective tissue disease, which include capillary hemorrhages (early stages), enlarged capillaries with lumen $>50$ $\mu \mathrm{m}$ (early stages), loss of capillaries, ramified capillaries, vascular architectural disorganization (advanced stages), and vascular areas (advanced stages) (Figure 4a-4c) [8]. Aspects of this pattern are seen in systemic sclerosis, systemic lupus erythematosus, and dermatomyositis [8]. However, significant dropout of capillaries in the setting of skin thickening of the fingers of both hands extending proximal to the metacarpal phalangeal joints is diagnostic for systemic sclerosis [9]. The presence of these microvascular changes add to the suspicion of Raynaud's phenomenon secondary to an underlying connective tissue disease and are helpful for serially examining a patient with increasingly severe Raynaud's phenomenon with a negative predictive value of $93 \%$ [8]. Interestingly, correlations are evident between the nailfold capillary morphology and clinical symptoms as well as severity of the disease in systemic sclerosis [8].

The importance of identifying nailfold capillaroscopy abnormalities are highlighted by its inclusion in the American College of Rheumatology (ACR) and European Union League Against Rheumatism (EULAR) criteria for the classification of systemic sclerosis [9], since nailfold capillaroscopy is established as a procedure that has high negative predictive value in normal findings and high specificity of the SSc pattern for the presence or development of SSc [8]. Importantly, nailfold capillaroscopy abnormalities in SSc patients predict mortality [10]. Additionally, nailfold capillaroscopy has a role to monitor disease activity and has been proposed as a biomarker [11].

\section{Laboratory Testing}

In a patient with Raynaud's phenomenon with abnormal capillaroscopy. Specific tests may assist in the identification of an underlying connective tissue disease $[1,12]$. In the setting of inflammatory arthritis, rash, and/or serositis an Antinuclear Antibody (ANA), complements C3 and C4, Urine Analysis (UA), Complete Blood Count (CBC), and a Smith antibody as well as a dsDNA antibody are warranted to further screen for SLE. If hand edema, pulmonary fibrosis, pulmonary arterial hypertension, or skin thickening is present then a centromeric antibody, anti-topoisomerase antibody (SCL 70), and RNA polymerase III antibody should be sent to investigate for SSc. Raynaud's phenomenon associated with significant sicca symptomatology, and small joint arthritis should prompt the evaluation of Sjogrens with the sending of a Rheumatoid Factor (RF), SSA and SSB antibody. An evaluation for dermatomyositis with a Creatine Kinase (CK) and Jo-1 antibody should be obtained in a patient presenting with rash which is heliotropic around the eyes, shawl-like on back, v-like on chest, and/or Gottron's papules associated with Raynaud's phenomenon. 
Citation: Frech TM, Breviu B, Zhu JT, Saketkoo L (2014) Nailfold Capillaroscopy: An Important Clinical Tool for the Vascular Surgeon. J Vasc Med Surg 2: 152. doi: 10.4172/2329-6925.1000152

Approximately 15 to 20 percent of patients with Raynaud's phenomenon who have autoantibodies, abnormalities of nail-fold capillaries, or both and who do not initially meet the criteria for a connective tissue disease ultimately will ultimately fulfill diagnostic criteria within two years [13]. Thus, if history is concerning for connective tissue diseases that are associated with Raynaud's phenomenon, such as SLE, SSc, Sjogrens syndrome, and dermatomyositis, these diseases can be screened for by sending appropriate laboratory tests if indicated.

\section{Conclusion}

The history of Raynaud's phenomenon is an important clinical symptom for the vascular surgeon to inquire about, particularly if critical digital ischemia is present. The use of capillaroscopy to routinely assess any patient complaining of Raynaud's phenomenon is imperative. The use of nailfold capillaroscopy by the vascular surgeon can help phenotype patients so that cost effective laboratory assessment is applied.

\section{References}

1. Wigley FM (2002) Clinical practice. Raynaud's Phenomenon. below N Engl J Med 347: 1001-1008.

2. Herrick AL (2005) Pathogenesis of Raynaud's phenomenon. below Rheumatology (Oxford) 44: 587-596.

3. Maricq HR, Weinrich MC, Keil JE, Smith EA, Harper FE, et al. (1989) Prevalence of scleroderma spectrum disorders in the general population of South Carolina. Arthritis Rheum 32: 998-1006.
4. Chatterjee S (2013) Q: When do Raynaud symptoms merit a workup for autoimmune rheumatic disease? below Cleve Clin J Med 80: 22-25

5. Silman A, Holligan S, Brennan P, Maddison P (1990) Prevalence of symptoms of Raynaud's phenomenon in general practice. below BMJ 301: 590-592.

6. Cutolo M, Sulli A, Smith V (2013) How to perform and interpret capillaroscopy. below Best Pract Res Clin Rheumatol 27: 237-248.

7. Baron M, Bell M, Bookman A, Buchignani M, Dunne J, et al. (2007) Office capillaroscopy in systemic sclerosis. below Clin Rheumatol 26: 1268-1274.

8. Cutolo M, Pizzorni C, Sulli A (2005) Capillaroscopy. below Best Pract Res Clin Rheumatol 19: 437-452.

9. Hoogen FVD, Khanna D, Fransen J, Johnson SR, Baron M, et al. (2013) classification criteria for systemic sclerosis: an American college of rheumatology/European league against rheumatism collaborative initiative. Ann Rheum Dis 72: 1747-1755.

10. Smith V, Riccieri V, Pizzorni C, Decuman S, Deschepper E, et al. (2013) Nailfold capillaroscopy for prediction of novel future severe organ involvement in systemic sclerosis. below J Rheumatol 40: 2023-2028.

11. Cutolo M, Smith V (2013) State of the art on nailfold capillaroscopy: a reliable diagnostic tool and putative biomarker in rheumatology? below Rheumatology (Oxford) 52: 1933-1940.

12. Chatterjee S (2010) Management of Raynaud's Phenomenon in the Patien with Connective Tissue Disease. below Curr Treat Options Cardiovasc Med 12: 185-204.

13. Spencer-Green G (1998) Outcomes in primary Raynaud phenomenon: meta-analysis of the frequency, rates, and predictors of transition to secondary diseases, below Arch Intern Med 158: 595-600. 